\title{
Preliminary Study on the Histology in the Head Sarcoma of Goldfish (Carassius auratus)
}

\author{
Lei Wang1, Zaizhong Chen1, Caixia Hu${ }^{1}$, Feng Yan², Jun $\mathrm{Xu}^{2}$, Xuehua Wang2, \\ Yuanyuan Zhou' ${ }^{2}$, Wei Wang ${ }^{2}$, Weihua Zhao ${ }^{2}$ \\ ${ }^{1}$ College of Fisheries and Life Sciences, Shanghai Ocean University, Shanghai, China \\ ${ }^{2}$ Shanghai Pudong Ornamental Fish Center, Shanghai, China \\ Email: chenzz@shou.edu.cn
}

Received 4 December 2014; accepted 31 December 2014; published 13 January 2015

Copyright (C) 2015 by authors and Scientific Research Publishing Inc.

This work is licensed under the Creative Commons Attribution International License (CC BY). http://creativecommons.org/licenses/by/4.0/

(c) (i) Open Access

\section{Abstract}

The sarcoma structure of goldfish was first analyzed by frozen section technique. The present study revealed the alkaline and acid phosphatase distribution in the head sarcoma of goldfish. And the histochemical staining in situ by calcium-cobalt method and lead nitrate method displayed a large amount of alkaline and acid phosphatases in the head sarcoma, with higher enzyme activity. The alkaline and acid phosphatases belong to hydrolases, which are widely present in various tissues. The results showed that the activity of external sarcoma is similar to the middle one, while there was more alkaline phosphatase near to the tissue sarcoma under the goldfish scales. And they have the strong activity. The study will lay the theoretical basis on enhancing the ornamental value of goldfish.

\section{Keywords}

\section{Goldfish, Head Sarcoma, Frozen Sectioning Method, AKP, ACP}

\section{Introduction}

It is known that the goldfish evolved from wild crucian carp [1] [2]. Compared with plants and other animals, goldfish has much more variation in color and body shape, which has great difference from the ancestor-wild crucian carp. The sarcoma of the goldfish is pathological tissue, which increased the burden on the head and gill covers, even can affect the fish's breathing. Sarcoma can be gradually increased with the growth process. For example, there is no sarcoma on the head of the hatched juveniles untill 3 - 4 months later. The rich head-sar- 
coma has been formed in many varieties of goldfish, such as White Oranda with Red Cap, Tigerhead Ranchu and so on. At present, although the goldfish with fleshy and beautiful head-sarcoma can be better bred, we have not clearly known well about the tissue and formation mechanism of them yet.

In the present study, the preliminary observation about alkaline and acid phosphatase had been determined by using frozen section technique. And the characteristics of the distribution about alkaline phosphatase and acid phosphatase on the sarcoma tissues during the Red Oranda had been observed.

The study can accumulate a certain theoretical basis for the future high-head goldfish sarcoma growth and development. It can even provide theoretical reference for further research goldfish sarcoma histological analysis as well as the formation mechanism of benign tumour.

\section{Materials and Methods}

\subsection{Sample}

The experimental goldfish Red Oranda was collected and analyzed in Aquarium Science Laboratory, Shanghai Ocean University. And they have the basically same specification and healthy body gesture.

\subsection{Histochemical Staining of Alkaline Phosphatase (AKP)}

The sarcoma cut from goldfish was embedded with embedding medium. The fresh tissue with $6 \mu \mathrm{m}$ thickness which was sectioned under low temperature was affixed to slides, and it was fixed for 10 minutes after drying. Acetone should be placed in $4^{\circ} \mathrm{C}$ refrigerator first. The slices were also carried out in the same environment. Alkaline phosphatase was incubated at $37^{\circ} \mathrm{C}$ in water baths. The slices were placed in alkaline solution $\left(37^{\circ} \mathrm{C}\right)$ for $15 \mathrm{mi}-$ nutes. Then the slices were washed by distilled water 2 - 3 times, each for 1 minute. The slices were put in the 2\% aqueous solution of cobalt nitrate for 5 minutes. The slices were washed by distilled water $2-3$ times, each for 1 minute, under the treatment of $1 \%$ sulfide aqueous solution for 1 minute. The slices were washed for 3 - 5 minutes by running water, washed by distilled water. Then the slices were airproof by neutral gel.

\subsection{Histochemical Staining of Acid Phosphatase}

The sarcoma extracted from goldfish was embedded with embedding medium. The fresh tissue with $6 \mu \mathrm{m}$ thickness which was sectioned under low temperature was affixed to slides, and it was fixed for 10 minutes after drying. Acetone should be placed in $4^{\circ} \mathrm{C}$ refrigerator first. The slices were also carried out in the same environment. Acid phosphatase was incubated at $37^{\circ} \mathrm{C}$ in water baths. The slices were placed in acid phosphatase solution $\left(37^{\circ} \mathrm{C}\right)$ for 30 minutes. Then the slices were washed by distilled water $2-3$ times, each for 1 minute. The slices were put in the $1 \%$ aqueous solution of glacial acetic acid for 30 seconds. The slices were washed by distilled water 2 - 3 times, each for 1 minute, under the treatment of $1 \%$ sulfide aqueous solution for 1 minute. The slices were washed for 3 - 5 minutes by running water, washed by distilled water. The slices were airproof by neutral gum.

The experiments were observed and photographed by inverted microscope named Japanese OLYMPUS IX71 and fluorescence microscope digital imaging system.

\section{Results}

\subsection{Distribution of ACP and AKP in Goldfish Sarcoma}

Through the frozen sectioning method, AKP was displayed with calcium-cobalt method as well as ACP displayed by lead nitrate method. After pigmentation, the position of AKP located in the sarcoma was the black particle or curdy precipitate (Figures 1-4). Furthermore, the position of ACP was the brown-yellow or darkbrown particles (Figure 5 and Figure 6). Anywhere the black cobalt sulfide was that can show the AKP was existed. Furthermore, we can estimate the activity of the phosphatase according to the depth of the color. For ACP, $\beta$-glycerol phosphate as substrate, it can release phosphate under the interpretation of ACP in the acidic $\mathrm{pH}$ of water. Phosphate combines with lead ion to form phosphate precipitation, and then replaced by sulfur ion, they produced the dark-brown lead sulphide precipitate.

Through the experimental observation, the two phosphatases are mainly distributed in the cell membrane (Figure 5(a)), and contain large amounts of protein particles (Figure 1 and Figure 5(a)). 


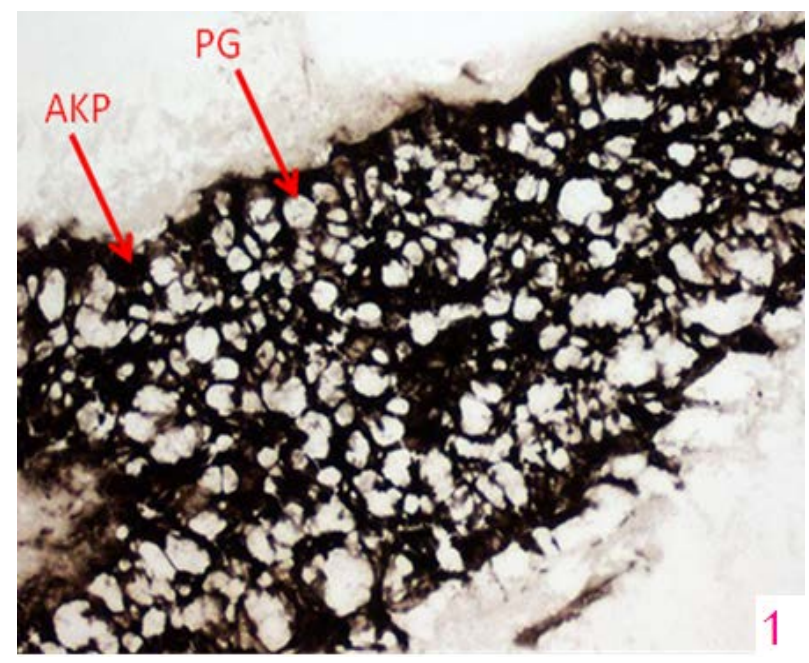

Figure 1. Transverse section of AKP pressing close to the tissue sarcoma near to the goldfish scales, $\times 10$.

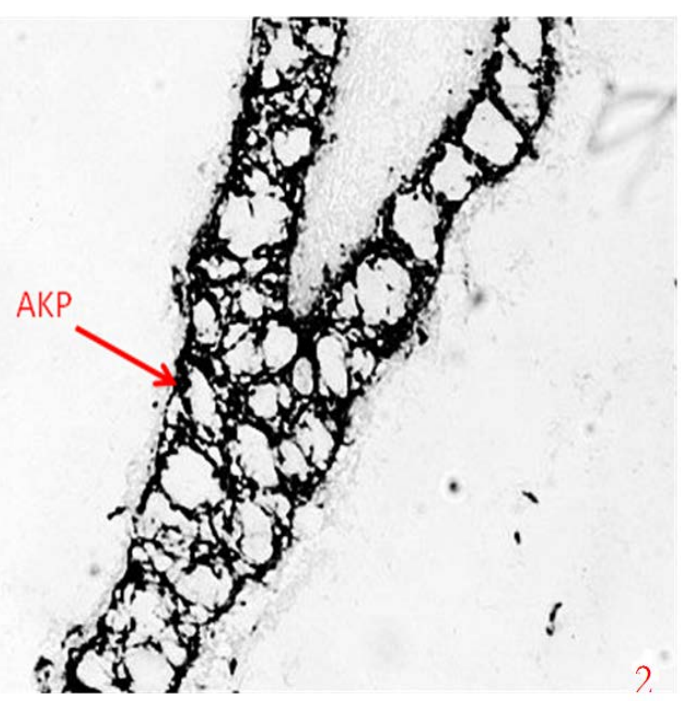

Figure 2. Transverse section of AKP of the external sarcoma, $\times 10$.

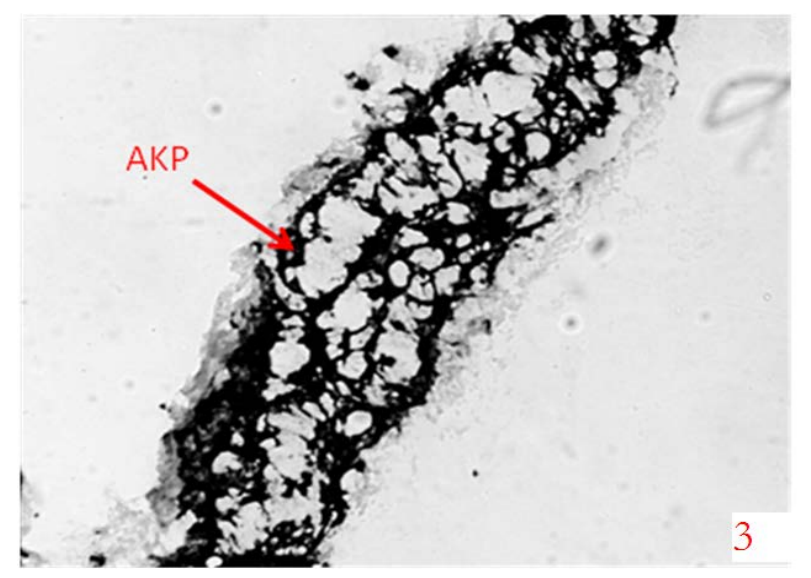

Figure 3. Transverse section of AKP of the middle location of sarcoma, $\times 10$. 


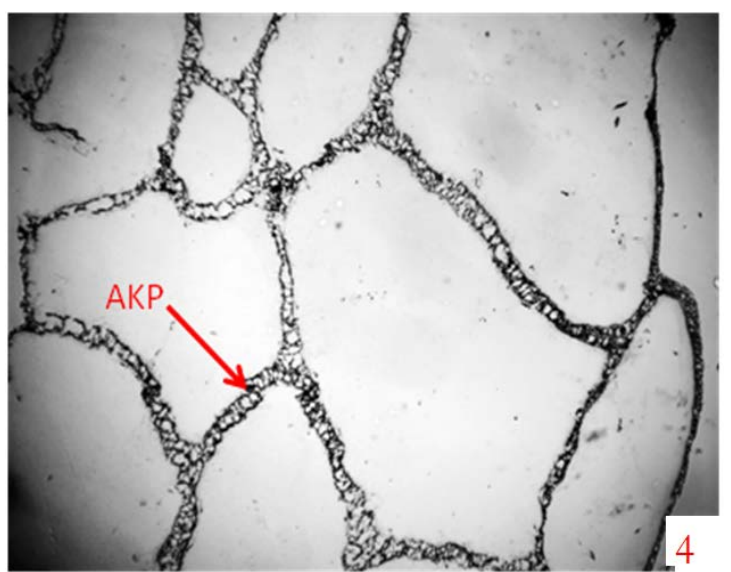

(a)

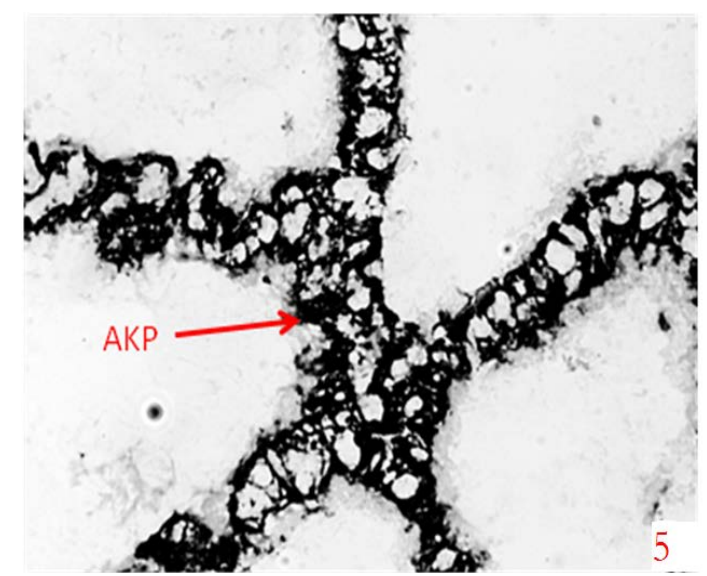

(b)

Figure 4. (a) (b) Longitudinal section of AKP on goldfish sarcoma, sequentially $\times 2, \times 10$.

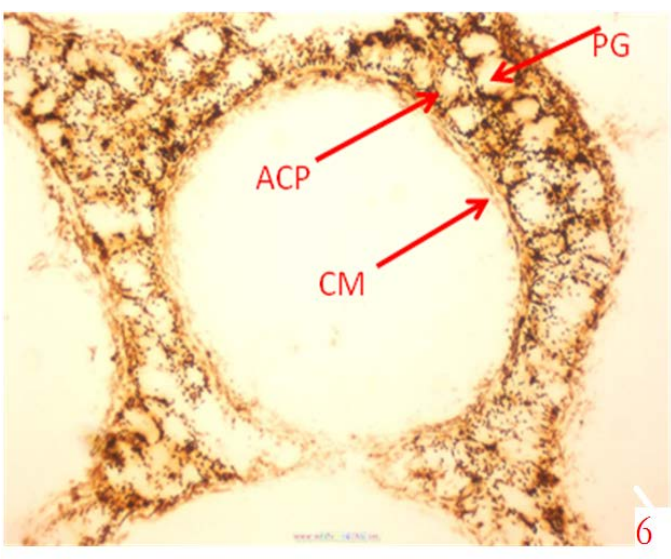

(a)

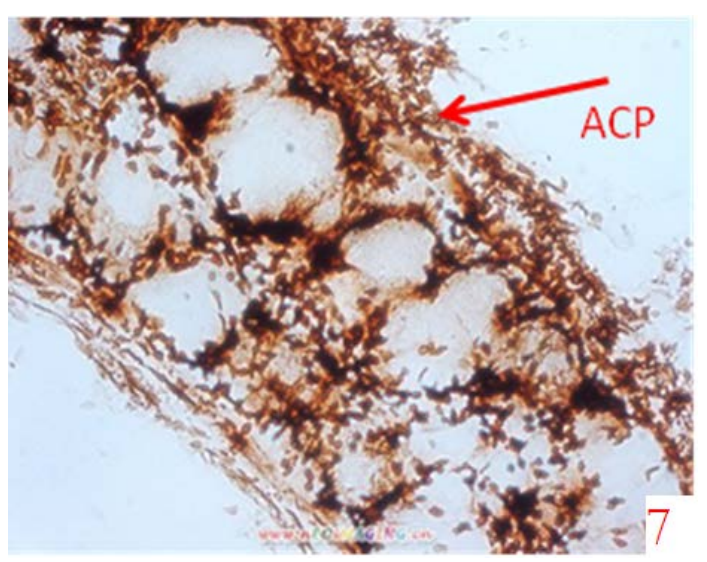

(b)

Figure 5. (a) (b) Transverse section of ACP on goldfish sarcoma, sequentially $\times 4, \times 20$.

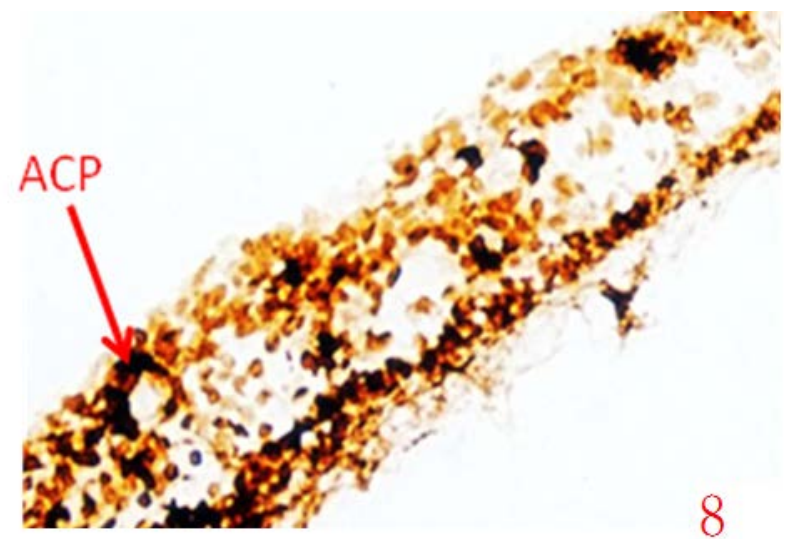

Figure 6. Longitudinal section of ACP on goldfish sarcoma, $\times 10$.

\subsection{Comparisons on Activity of AKP and ACP in Different Sectioning Parts of Sarcoma}

In the present study, the AKP and ACP was not only sectioned transversely, but also done longitudinally. It shown that the activity of AKP and ACP in the upper part of sarcoma was similar to the middle one, while there 
was more AKP pressing close to the tissue sarcoma near to the goldfish scales. And they have strong activity. The detailed information was shown in Figures 1-3.

\section{Discussion}

\subsection{AKP and ACP Localization in the Head-Sarcoma of Red Oranda}

AKP is a hydrolase enzyme responsible for removing phosphate groups from many types of molecules, including nucleotides, proteins, and alkaloids. The optimal $\mathrm{pH}$ for the activity of the $E$. coli enzyme is 8.0, while the bovine enzyme optimum $\mathrm{pH}$ is slightly higher at 8.5. AKP played a clear promoting role in the calcium metabolism to the mussel Hyriopsis [3].

ACP is a hydrolytic enzyme, mainly located in lysosomes in cells, which is a sign of lysosomal enzymes. It was existed in the macrophages, and located within the lysosome.

ACP appeared in the lysosome, nucleus, cell membrane, mitochondria of various organizations in the body of Nabin prawn. AKP appeared in the cell membrane, nucleus, lysosome, endoplasmic reticulum of the organization, even in the lipid droplet and the microvilli surrounding the liver tissue [4]. Phosphate combines with lead ion to form Phosphate precipitation, and then replaced by sulfur ion, they produced the drak-brown lead sulphide precipitate [5].

During the experiment operation process, the fluid concentration of lead ion is essential, even must be prepared currently. It will lead to diffusion while low concentration of lead ions caused that the phosphate ions cannot be completely captured. And may result in the false-positive nuclear staining by entering the nucleus, it may also lead to the phenomenon of enzyme-proliferation and nuclear staining due to long time of incubation.

\subsection{Role of ACP and AKP during the Formation of Sarcoma}

Both AKP and ACP are belonged to the phosphomonoesterase. They widely exist in a variety of animals [6]-[9]. They can not only catalyze phosphate monoester hydrolysis, but also involve in transfer of phosphate groups directly. They both are important detoxification system in animals and play an important role in the process of ossification in the animal organism and phosphide, as well as the digestion, absorption and transport in other nutrient digestion. Meanwhile, as the main parts of animal lysosomal enzymes, ACP and AKP play a role in the immune response. The study on the ACP and AKP to the haemolymph of Chlamys farreri indicated that the lysosome was a strong positive ACP, AKP-positive granules distributed along the cell membrane [10]. Both biological macromolecules and intracellular organelles have a certain life span, and the lysosomes can surround their own aging and excess organelles to form autophagic body. Useful materials can be still reabsorption and utilization after digesting the organelle. Lysosomal enzymes can also remove temporarily unwanted enzyme or some metabolites [11].

The goldfish can still survive and grow new sarcoma after sarcoma's excision through the latter observation. It can suspect that may be due to the existence of a large number of phosphatase, and have very strong activity.

\subsection{Attentions to Frozen Sectioning Method in Operation}

The temperature regulation is very important during the experimental operation. Illsuited temperature may make the experiment failed, and it will be difficult to cut into slices. Furthermore, it is easy make tissue damage, affects the final result. The time that ammonium sulfide impregnated it should be controlled well. Otherwise, it will affect the observation result. The blade should be sharp and we should choose the accurate parts across the board. However, it must avoid unnecessary stretching and compression.

The test should be repeated in order to find the best operating temperature for goldfish sarcoma slice, and to maintain activity, it must use acetone before $4^{\circ} \mathrm{C}$ to fix it. The water washing the slice should be gently, otherwise it will result the organization in shedding. In addition, due to the volatilization, the ammonium sulfide solution should be prepared simultaneously.

\section{Acknowledgements}

This work was funded by the Key Project of Developing Agriculture through Science and Technology of Shanghai Municipal Agricultural Commission Shanghai, Shanghai University Knowledge Service Platform-Shanghai 
Ocean University Aquatic Animal Breeding Center (ZF1206) and the Shanghai Leading Academic Discipline Project (Grant No. Y1101). And we would like to thank laboratory members for their encouragement and meaningful discussions.

\section{References}

[1] Liang, Q.J. (1995) The Origin and Evolution of Goldfish. Biology Bulletin, 30, 14-16.

[2] Liang, Q.J., Peng, Y.X. and Yu, Q.M. (1998) The Judgment Analysis and Cluster Analysis between Wild Crucian Carp and Five Varieties of Goldfish. Journal of Aquatic Biology, 23, 236-242.

[3] Shi, Z.Y., Li, Y. and Xie, X.Z. (2008) Relations between Alkalinity Phosphatase and Calcium Metabolism in Mantle of Hyriopsis cumingii. Journal of Shanghai Fisheries University, 17, 291-297.

[4] Sun, J.Q., Xu, Y., Zhang, H.Q. and Ye, J.Q. (2007) Study on Cytochemical Location of Acid Phosphatase and Alkaline Phospatase of Litopenaeus vannamei. Journal of Fudan University (Natural Science), 46, 947-951.

[5] Wang, J.F. and He, Y.M. (2004) Experiment of Cell Biology. Science Press, Beijing, 61-62.

[6] Wang, H.Y. (2000) Atlas of Chinese Goldfish. Science Press, Beijing, 2-6.

[7] Zhang, W., Su, Y.Q., Wang, J., Quan, C.G. and Liang, J.R. (2001) Biochemical Composition of Five Common Reared Fishes. Marine Science Bulletin, 20, 26-31.

[8] Wang, A.L., Liu, J.H. and Wang, W.N. (2005) Comparative Studies on the Total Pigment and Pigment Components of Color Carp. Acta Hydrobiologica Sinica, 29, 694-698.

[9] Spinelli, J. and Mahnken, C. (1978) Carotenoid Deposition in Pen-Reared Salmonids Fed Diets Containing Oil Extracts of Red Crab (Pleuroncodes planipes). Aquaculture, 13, 2l3-223. http://dx.doi.org/10.1016/0044-8486(78)90003-0

[10] Sun, H.S. and Li, G.Y. (1999) The Activities and Their Electron Microscopic Cytochemistry Study of AKP and ACP in Haemolymph of Chlamys farreri. Journal of Fishery Sciences of China, 6, 6-9.

[11] Pan, D.R. (2007) Cell Biology. Science Press, Beijing, 136-137. 
Scientific Research Publishing (SCIRP) is one of the largest Open Access journal publishers. It is currently publishing more than 200 open access, online, peer-reviewed journals covering a wide range of academic disciplines. SCIRP serves the worldwide academic communities and contributes to the progress and application of science with its publication.

Other selected journals from SCIRP are listed as below. Submit your manuscript to us via either submit@scirp.org or Online Submission Portal.
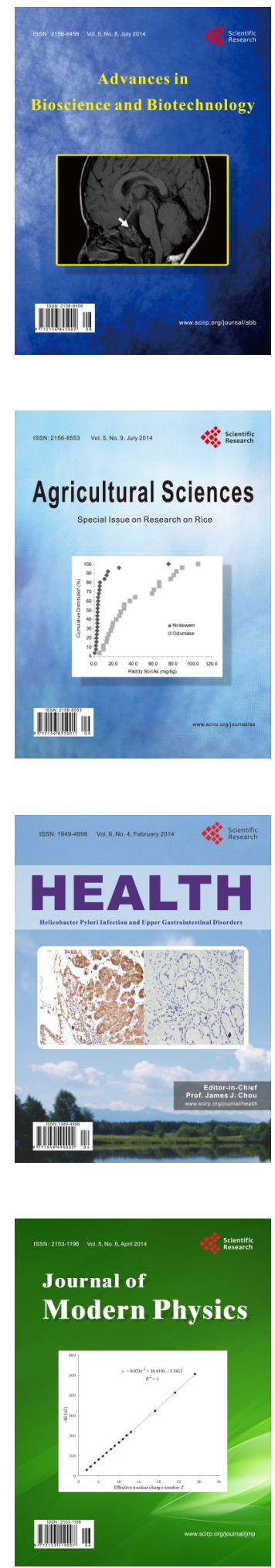
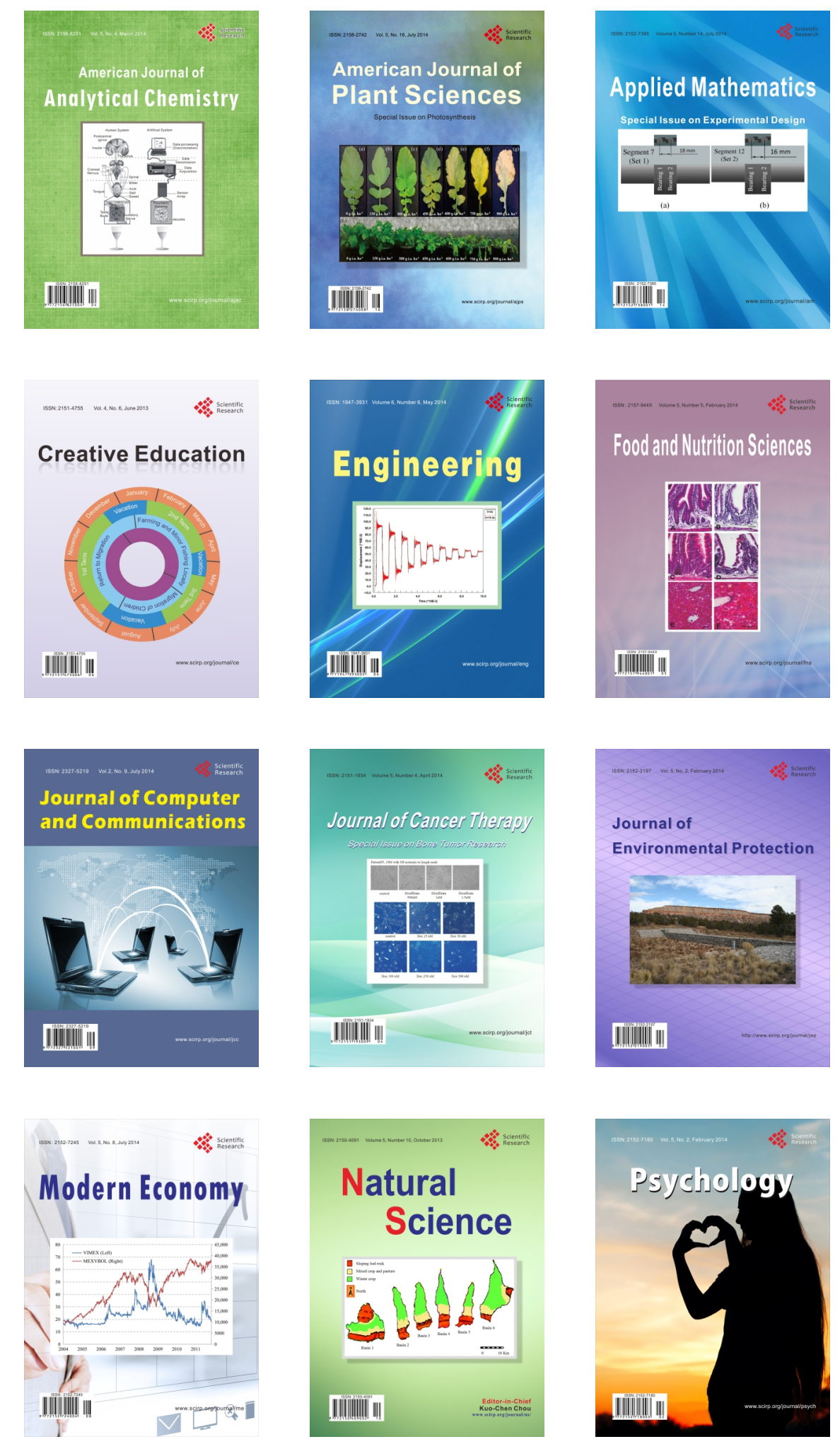